\title{
Determination of source parameters using broad-band seismograms
}

\author{
Laura Beranzoli, Domenico Giardini and Enzo Boschi \\ Istututo Nazionale di Geofisica, Roma, Italia
}

\begin{abstract}
The capability of a regional network to provide an accurate description of seismic source is tested estimating the focal mechanism, hypocentral depth, seismic moment and source time function of some large regional and teleseismic events recorded at MEDNET. The procedures we developed to this aim perform surface waves and body waves modelling and include synthetic seismograms computation based on the Free Oscillation Theory and Ray Theory. A comparison between the results obtained and the ones provided by other global seismic network data centers shows the high accuracy reached in the estimate of the principal source parameters when
high-quality data are available.
\end{abstract}

\section{Introduction}

MEDNET is the very broad-band seismographic network installed by the Istituto Nazionale di Geofisica in countries of the Mediterranean area, with the final goal of 12 stations and a spacing of about $1000 \mathrm{~km}$ between stations. Started in 1987, the project is motivated both by research interest and by seismic-hazard monitoring.

The network has been designed following the highest technical standards: STS-1/VBB sensors, Quanterra 24 bits A/D converters and $140 \mathrm{~dB}$ Dynamic range. Seven sites are now operating in Italy and Northern Africa countries.

The MEDNET Data Center in Rome takes care of data collection, verification, quality control, archival and distribution, monitoring of station performance.

To test the capability of a regional network to provide an accurate description of seismic source, we developed a waveform modelling approach which combines long-period body and surface waves to derive the moment tensor, and broad-band $P$ displacement pulses to model the source time function and constrain depth.

\section{Long-period waveform modelling}

The inversion for the moment tensor is based on the following elements:

i) we use 6 hours of three-component records, containing long-period body waves and the first 3 or 4 orbits of Rayleigh and Love waves;

ii) synthetic seismograms are generated by summation of normal modes at periods exceeding 100 seconds, computed for model PREM of Dziewonski and Anderson (1981) using a scheme by Woodhouse (1988); complete seismograms include fundamental modes and overtones, and model both body and surface waves; for a rapid analysis of shallow events the summation can be restricted to fundamental modes and to the synthesis of surface waves;

iii) inversion is performed in a narrow frequency band $((5 \div 7) \mathrm{mHz})$ for a fixed hypocentral location; a weighting scheme is introduced where each trace is weighted by its norm; the moment tensor is constrained to have vanishing trace or equivalently null isotropic component (following Dziewonski et al., 1981);

iv) a time correction is computed for each 
trace after the inversion for the moment tensor; the source half-duration is estimated as the time correction common to all traces; the residual time corrections account for hypocentral mislocations, spatial extent of the source and mantle heterogeneities;

v) depth is retrieved by variance minimization on different trials (Romanowicz and Suarez, 1983);

vi) a constraint involving the minimization of the moment tensor norm, weighted by a parameter $\varepsilon$ (see, e.g., Menke, 1984) is imposed; by varing the parameter $\epsilon$ it is possible to explore the uncertainty and resolution associated with individual elements of the moment tensor.

\section{Broad-band waveform modelling}

We use broad-band modelling to constrain the fault geometry and evaluate the source time function and depth. The modelling is based on the following elements:

i) stations in the $(30 \div 90)^{\circ}$ distance range are used; synthetic seismograms are generated by classic Ray-Theory (Langston and Helmberger, 1975 ) et each station site assuming a point source model and defining a horizontally layered structure;

ii) the focal mechanism is given by a longperiod analysis; the seismic moment obtained from long-period analysis is considered as a bound on the source time function integral (cf. Ekström, 1989);

iii) we invert for the source time function to model $P$ and $S H$ displacement pulses in shape and amplitude; the source time function is thought to be composed by triangular source time elements of $(2 \div 4) \mathrm{s}$ duration displaced by $(1 \div 2)$ s to each other (Nabelek, 1984); a positivity constraint for the source time function is imposed in the inversion;

iv) depth is evaluated by trial-and-error modelling of the surface reflections $p P$ and $s P$;

vi) attenuation is introduced with a $t^{*}$ of 1 second for $P$ waves and an initial $t^{*}$ of 4 seconds for $S H$ waves (Nabelek, 1984); $t^{*}$ can be adjusted for $S H$ waves to obtain the same shape and duration of the source time function for both $P$ and $\mathrm{SH}$ waves.

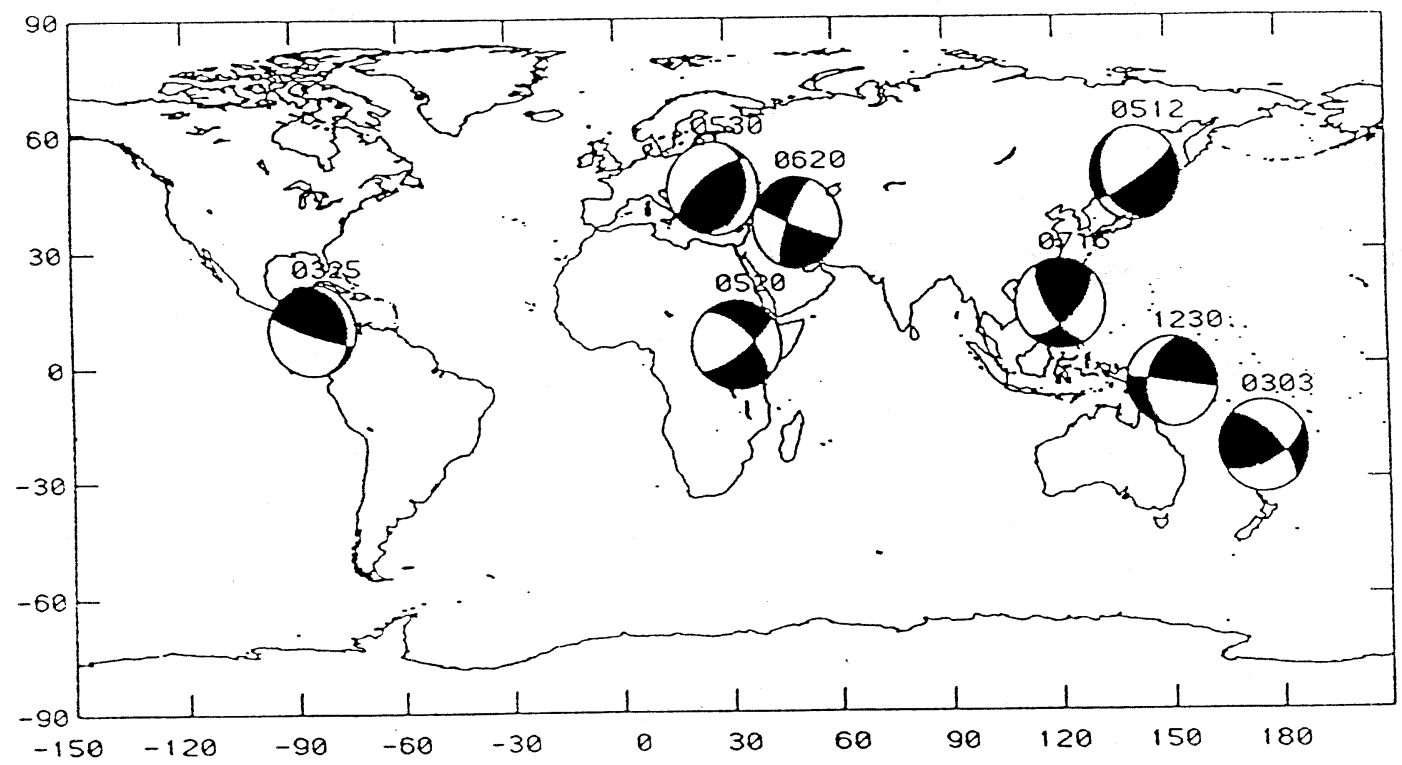

Fig. 1. The moment tensor inversion procedure was applied to the MEDNET recordings of large 1990 events reported in this map together with the focal mechanisms and month and day of occurrence; among the analysed events the 20 June Iran, 30 May Romania and 16 July Philippine caused heavy casualties. 


\section{Experiments}

Moment tensor inversion for some of the largest 1990 events has been performed.

The locations of the examined events are reported in the map of fig. 1 together with the corresponding month and day of occurrence and the focal mechanisms, obtained using the method prevously described.

We retrieve a comparison between the source geometry and seismic-moment estimate and the one derived from global seismic networks and different research groups distributed by NEIC is reported in fig. 2; in almost cases both seismicmoment estimates and focal mechanisms derived from MEDNET data using the procedures described in this study agree with the Centroid Moment Tensor solution (CMT) pointing out the capability of a regional seismological network to provide an accurate description of the rupture processes.

\begin{tabular}{cccccccc}
\hline \hline & Date & Region & $m_{b}$ & MEDNET & CMT & Ppt & Usgs \\
\hline A & $90 / 03 / 03$ & Fiji & 6.3 & $2.8 \times 10^{27}$ & $3.0 \times 10^{27}$ & $4.0 \times 10^{27}$ & $1.3 \times 10^{27}$ \\
B & $90 / 03 / 25$ & Costa Rica & 6.2 & $7.7 \times 10^{26}$ & $1.1 \times 10^{27}$ & $6.0 \times 10^{26}$ & - \\
C & $90 / 05 / 12$ & Sakhalin & 6.5 & $7.0 \times 10^{26}$ & $8.2 \times 10^{26}$ & $6.0 \times 10^{27}$ & $7.9 \times 10^{26}$ \\
D & $90 / 05 / 20$ & Sudan & 6.7 & $5.0 \times 10^{26}$ & $5.3 \times 10^{26}$ & $1.6 \times 10^{27}$ & $7.4 \times 10^{26}$ \\
E & $90 / 05 / 30$ & Romania & 6.7 & $2.7 \times 10^{26}$ & $3.0 \times 10^{26}$ & $7.0 \times 10^{26}$ & - \\
F & $90 / 06 / 20$ & Iran & 6.4 & $1.1 \times 10^{27}$ & $1.4 \times 10^{27}$ & $2.0 \times 10^{27}$ & $1.1 \times 10^{27}$ \\
G & $90 / 07 / 16$ & Luzon (Philippine) & 6.5 & $4.6 \times 10^{27}$ & $4.1 \times 10^{27}$ & $8.0 \times 10^{27}$ & - \\
H & $90 / 12 / 30$ & New Britain & 6.7 & $1.9 \times 10^{27}$ & $1.8 \times 10^{27}$ & $8.0 \times 10^{26}$ & $2.0 \times 10^{27}$ \\
\hline
\end{tabular}

A<smiles>CCCCCCCCCCCCCCCCCCCC</smiles>

B

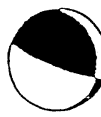

C

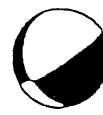

D

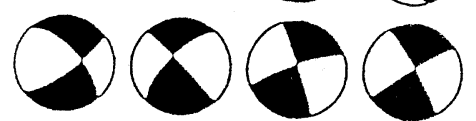

$E$

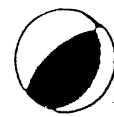

F
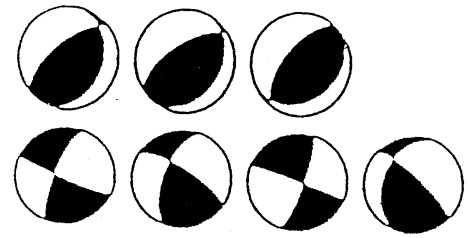

G

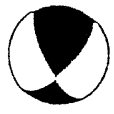

$\mathrm{H}$
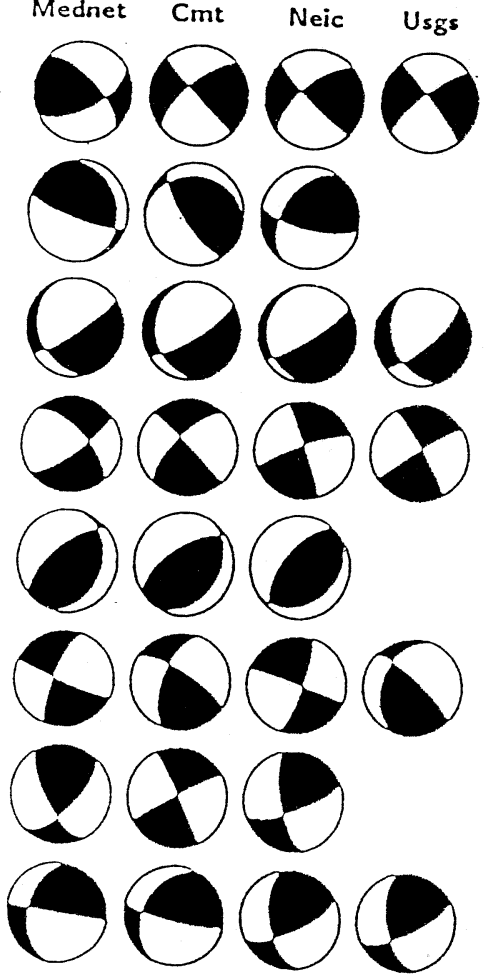

Fig. 2. The top table reports a comparison of the seismic-moment estimates in dyne $\cdot \mathrm{cm}$ produced by MedNeT, U.S. Geological Survey (Usgs), Papeete Observatory research group (Ppt) together with the Centroid Moment Tensor solution (CMT). A comparison of the focal mechanisms of the same agencies and research groups is shown in the bottom panel.

The long-period waveform modelling for the inversion of the moment tensor relative to the Sakhalin and Iran events is shown in fig. 3. In the left windows are reported filtered data (continuous line) and synthetic seismograms (dashed line) for the VSL and MDT MEDNET stations, respectively; corresponding frequency band spectra are on the right. The frequency band inside which the inversion has been performed is shown in bars $((5 \div 7) \mathrm{mHz})$.

In a second experiment we use long-period and broad-band waveform modelling to explore uncertainties in source parameters of May 20 Sudan event. We invert the best 36 long-period filtered traces for 16 Global Digital Seismic Network and MEDNET stations, characterized by excellent signal-to-noise ratio and simple, uncontaminated surface-wave trains. The results of five 

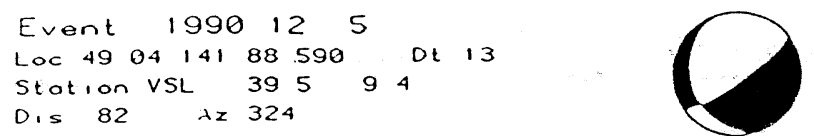

Vor 220

Froa 0.005000070

ScMom a $70 E+27$
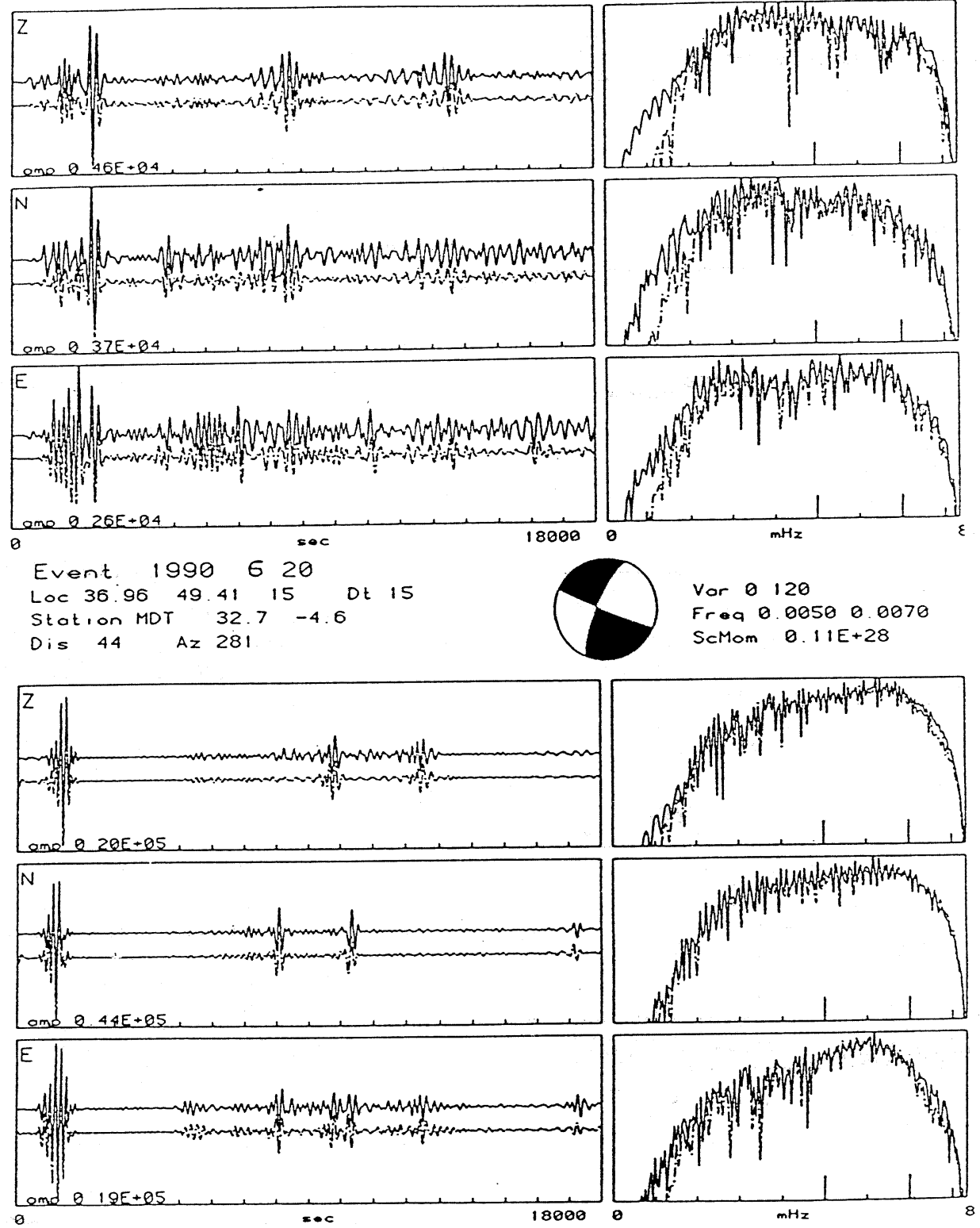

Fig. 3. In the left windows are reported filtered data (continuous line) and synthetic seismograms (dashed line) for the VSL and MDT MEDNET station for the Sakhalin and Iran events respectively; corresponding frequency band spectra are on the right. The frequency band inside which the inversion has been performed is shown in bars $((5 \div 7) \mathrm{mHz})$. 
a)

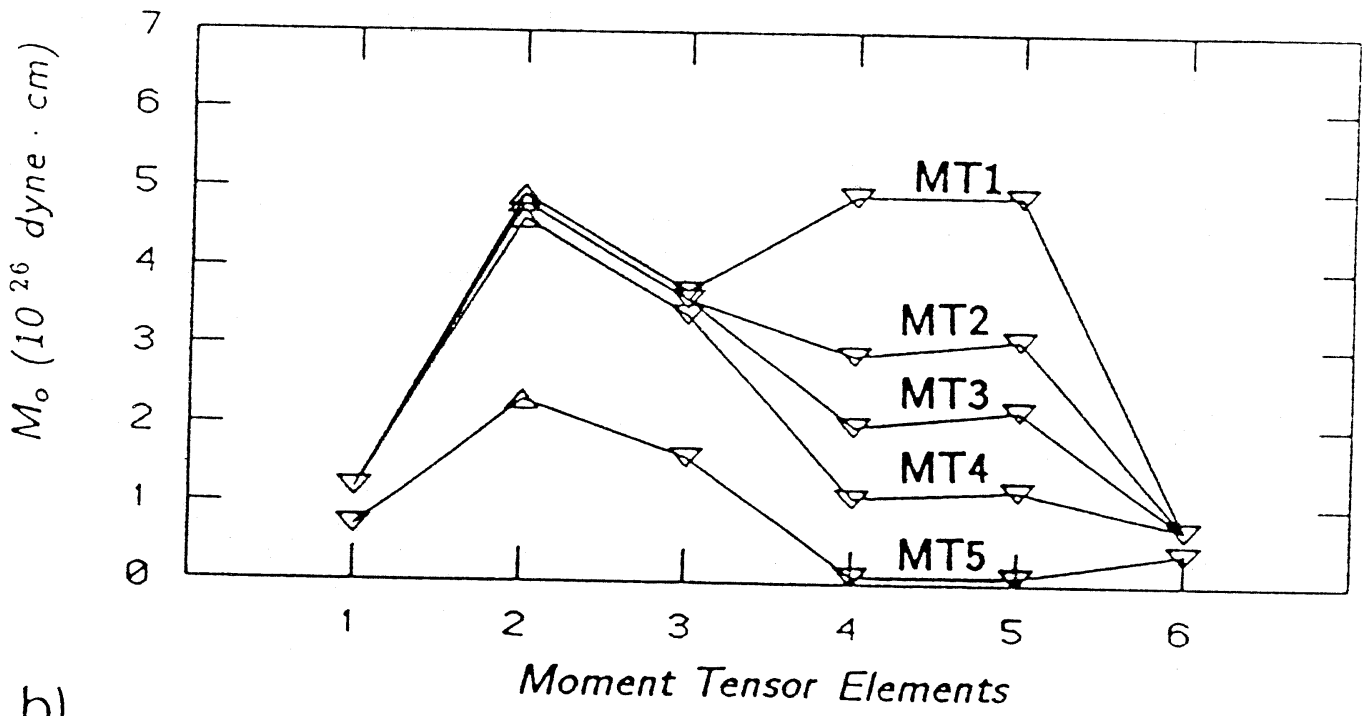

b)
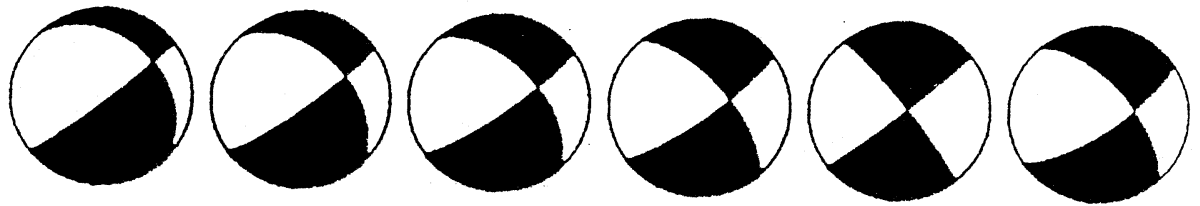

\begin{tabular}{ccccccc}
\hline & MT1 & MT2 & MT3 & MT4 & MT5 & MN \\
\hline$\varepsilon$ & 0.00 & 0.01 & 0.02 & 0.05 & 1.0 & 0.02 \\
$R$ & 0.213 & 0.215 & 0.218 & 0.225 & 0.449 & 0.174 \\
$M_{0}$ & 8.2 & 6.1 & 5.3 & 4.5 & 2.0 & 5.0 \\
$f p l-1$ & $313 / 32$ & $315 / 45$ & $316 / 53$ & $317 / 65$ & $320 / 85$ & $316 / 62$ \\
$f p l-2$ & $51 / 85$ & $53 / 81$ & $53 / 79$ & $52 / 79$ & $50 / 86$ & $55 / 73$
\end{tabular}

Fig. 4. a) Amplitude of the six elements of the moment tensor for five inversions performed for 20 May Sudan event with different minimization contraint (MT1 to MT5); b) fault mechanism and inversion parameters $R$ and the fault plane geometry (strike, slip angles) moment $M_{0}$ (in dyne $\cdot \mathrm{cm}$ units), the normalized variance data.

inversions (MT1 to MT5) for the six elements of the moment tensor using dumped least-square technique are shown in fig. 4a). By varying the parameter $\varepsilon$ it is possible to explore the uncertainty and resolution associated with the individual elements of the moment tensor. As it is common for shallow events (cf. Ekström, 1989) we observe a larger instability associated with the
$M_{r \theta}$ and $M_{r \phi}$ : a non-constrained solution (MT1) has a large seismic moment of $12 \times 10^{26}$ dyne $\mathrm{cm}$, dominated by the $M_{r \theta}$ and $M_{r \phi}$ components, and reaches a good normalized variance of $R=$ 0.277 . By increasing the contraint $\varepsilon$, those components decreased significantly, and so does the seismic moment, while the other seismic elements remain unchanged and the variance in- 


\section{May 201990 Sudan Earthquake}

$M_{0}=5.3 \times 10^{26}$ dyne $\cdot \mathrm{cm}$

$h=9 . \mathrm{km}$
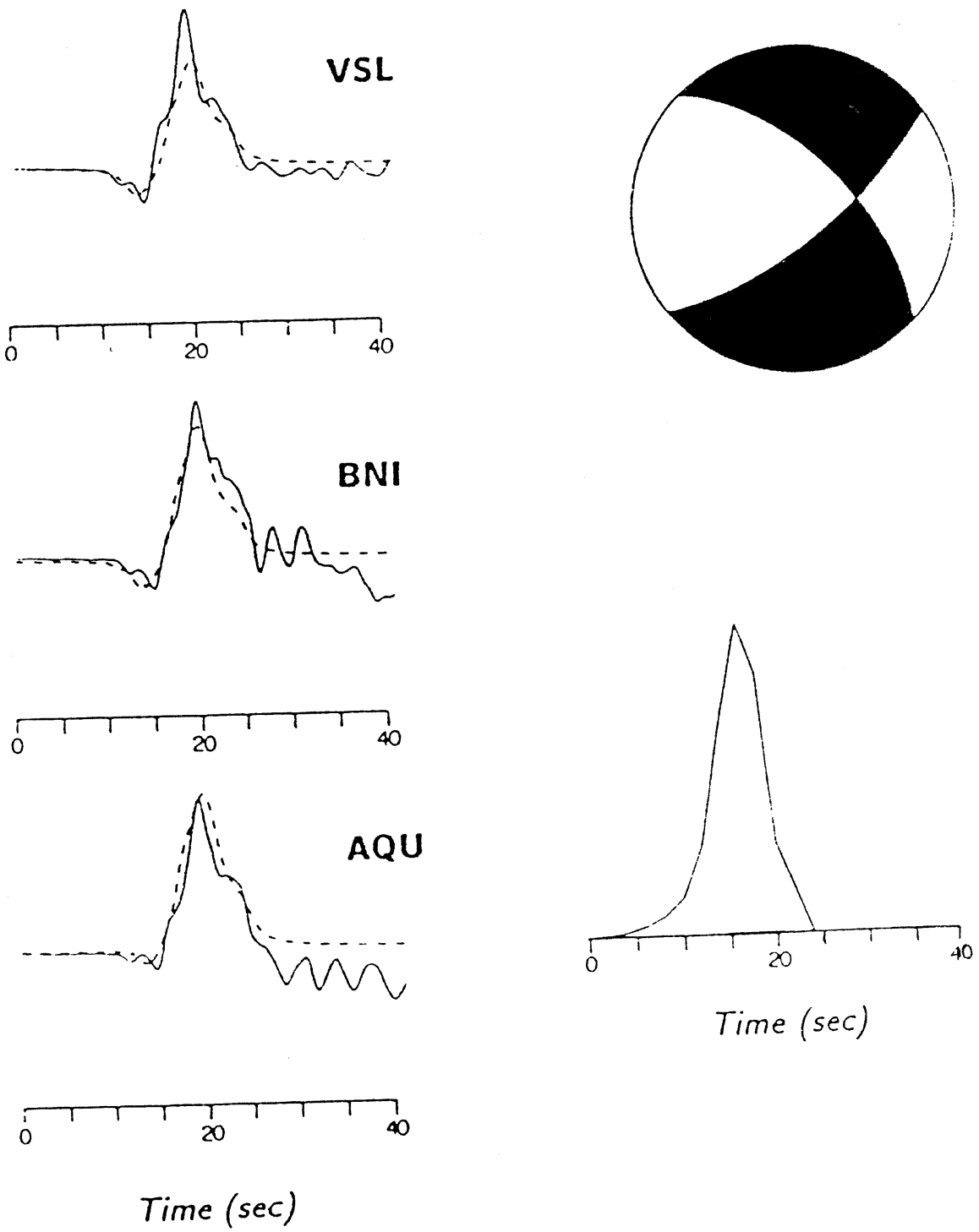

Fig. 5. $P$ waveform modelling at the three MEDNET stations telemetered to Rome in real time for the May 20 Sudan event: BNI, AQU and VSL. The preferred mechanism is solution MN of fig. 4 obtained by inversion of long-period MEDNET data. The sorce time function is also shown. A depth of $(10 \div 12) \mathrm{km}$ is needed to produce a successful fit. 
creases only slightly. It is only with a very strong constraint $(\varepsilon=1.0)$ that the solution (MT5) degrades considerably $(R=0.549)$, and all moment tensor elements are reduced $\left(M_{0}=1.8 \times 10^{26}\right.$ dyne $\cdot \mathrm{cm})$.

The geometry of focal mechanism reflects the proportions of the moments tensor elements (fig. 4b)); unconstrained solutions are dominated by the dip-slip elements $M_{r \theta}$ and $M_{r \phi}$ whereas more constrained tensors show a strike-slip geometry.

The solution indicated as MN in fig. 4b) is obtained using only MEDNET data and is quite similar to the MT3 and MT4 solutions obtained from global data, showing that even with few stations from a high-quality array it is possible to evaluate in real-time significant earthquakes (Ekström et al., 1986).

We turn to broad-band modelling to constrain the fault geometry and to evaluate the source time function and depth. Again we test the capability of the MEDNET regional array to describe the source process of teleseismic events by modelling $P$ pulses for the three MEDNET stations telemetered to Rome: BNI, AQU and VSL. We use the focal mechanism MN in fig. 4, obtained by inversion of long-period MEDNET data.

The results of modelling are shown in fig. 5 together with source time function. The early part of the signals, containing the contribution of the source, is well modelled on all traces; the later portion shows high-frequency ondulations which are different for each station and are produced by reverberations under the station.

It is worth noting that the source time function models all coherent details of the waveforms, produced by source complexity, but is not able to reproduce the structural effects. The source time function shows an almost symmetrical shape characterized by a fast starting in the rupture process and a fast stopping. A depth of

a)

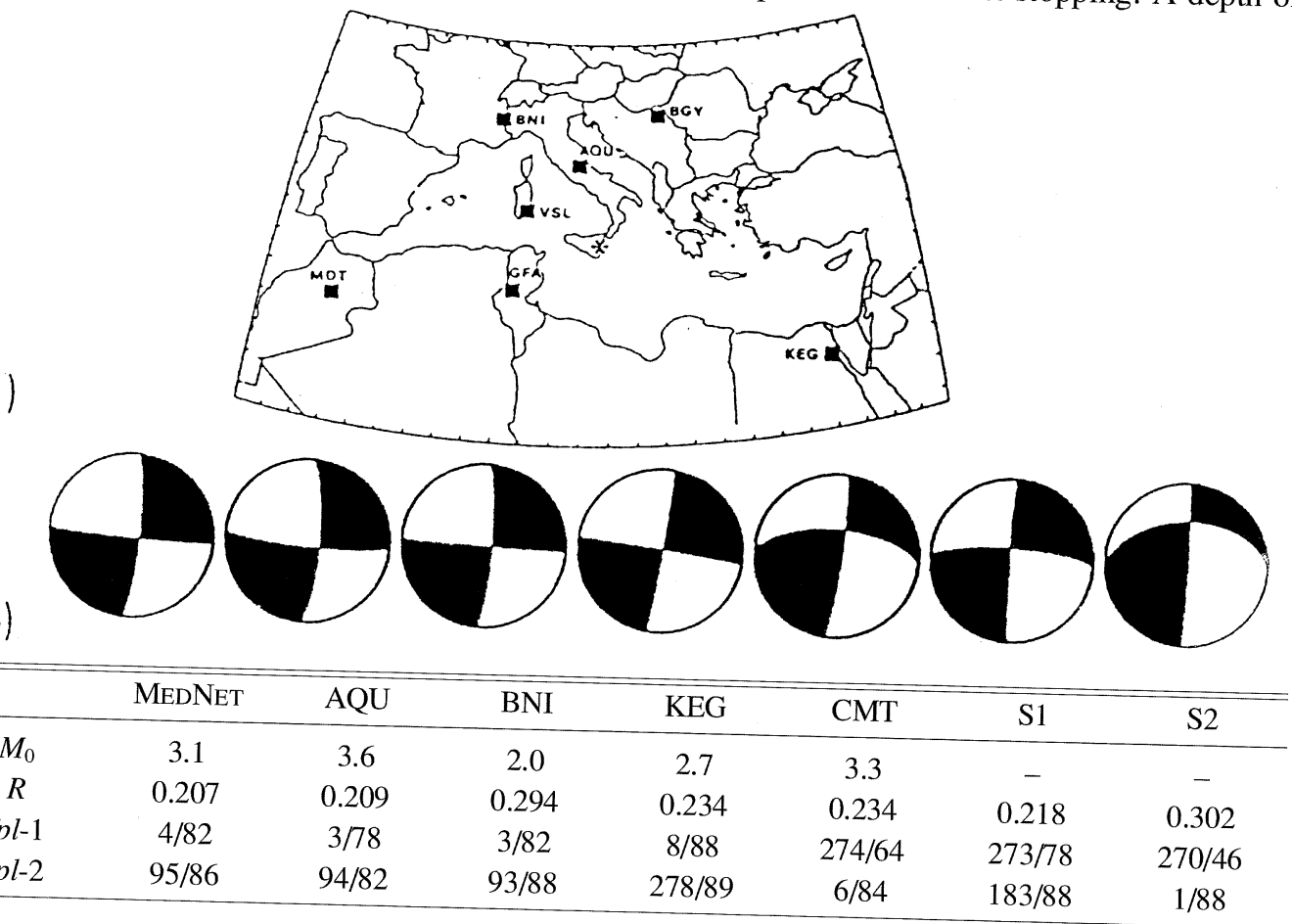

b)

Fig. 6. a) MEDNET stations distribution and December 13 Eastern Sicily event epicenter (asterisk); b) results of multiple MEDNET stations and single station (AQU, BNI, KEG) data inversion for moment tensor in comparison with CMT solution and first motion solutions (S1, Amato, S2, Gasparini, personal communications). Seismic-moment estimation, normalized variance, and focal planes orientation are also reported for each solution. 

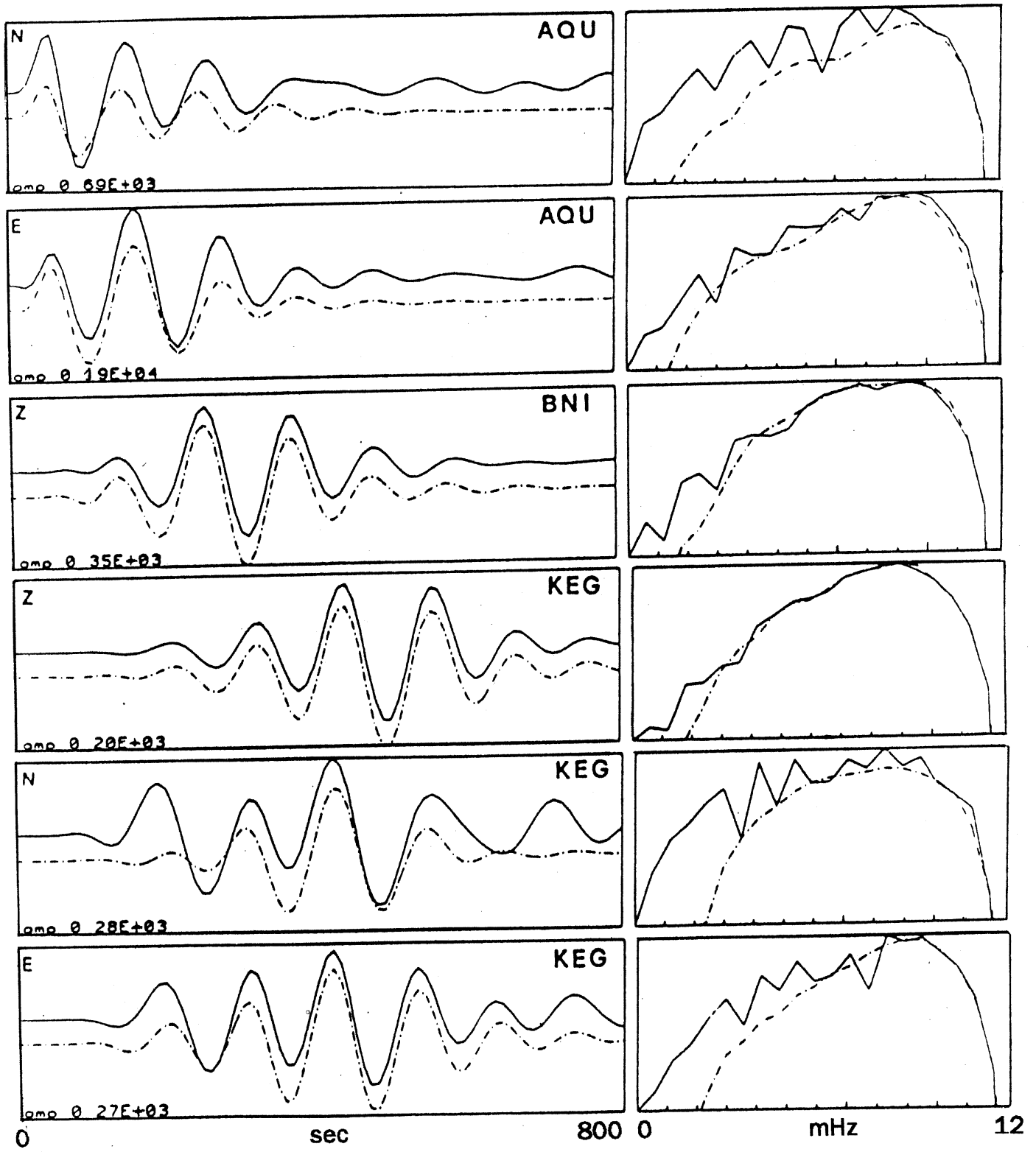

Fig. 7. Long-period waveform modelling: in the left windows are filtered data (continuous line) and synthetic seismograms (dashed line) for the indicated stations and components; corresponding frequency spectra are on the right. The frequency band included in the inversion ranges 7 to $10 \mathrm{mHz}$. 
$(10 \div 12) \mathrm{km}$ is needed to produce a successful fit.

In a third experiment, long-period waveform inversion for moment tensor has been performed for regional events. Results obtained for 1990 , December 13 Eastern Sicily event $\left(m_{b}=5.5\right.$, $M_{s}=5.3$, NEIC) are shown in comparison with the ones proposed by other Authors. The distribution of the MEDNET stations with respect to the epicenter, as shown in fig. 6a), points out a small azimuthal gap.

The table in fig. 6b) reports the results produced by inverting both MEDNET data jointly and data from single station. The $\mathrm{S} 1$ and $\mathrm{S} 2$ solutions have been obtained by first motion analysis.

All the focal mechanisms are characterized by the predominance of the trascurrence component with a slight variation in E-W focal plane dip. Large variation in the estimate of seismic moment $M_{0}$ in $10^{17} \mathrm{~N} \cdot \mathrm{m}$ is observed only when single-station inversion is performed while the CMT solution and the one retrieved using all MEDNET data available provide almost the same seismic moment.

The long-period waveform modelling of some MEDNET stations recordings is reported in fig. 7. The frequency band included in the inversion ranges 7 to $10 \mathrm{mHz}$. Even if the N-S components of AQU and KEG stations are better modelled in phases than in amplitudes, the modelling is quite satisfactory.

\section{Conclusions}

The description of the source geometry provided by high-quality data from a regional network appears in good agreement with the results from global network data. The long-period waveform modelling technique described is a powerful tool in focal mechanism determination and gives the needed information to retrieve source time function and depth from broad-band waveform modelling. The comparison of the estimates of seismic moment derived, for all the events studied, from MEDNET data with the ones from other agencies and authors, shows negligible disagreements caused by the different methodological approaches and data set.

\section{Acknowledgements}

We are grateful to the MEDNET Data Center and to the NEIC who make available the digital seismic data needed for this research. We thank Prof. Enzo Boschi for his leading role in the development of MEDNET and his continuous support.

\section{REFERENCES}

DZIEWonski, A.M. and D.L. ANDERSON (1981): Preliminary Reference Earth Model (PREM), Phys. Earth Planet. Inter., 25, 297-356.

DZIEWONSKI, A.M., T.A. CHOU and J.H. Woodhouse (1981): Determination of earthquake source parameters from waveform data for studies of global and regional seismicity, J. Geophys. Res., 86, 2825-2852.

EKSTRÖM, G.A. (1989): A very broad band inversion method for the recovery of earthquake source parameters, Tectonophysics, 166, 73-100.

EKSTRÖM, G.A., A.M. DZIEWONSKI and J.M. STEIM (1986): Single station CMT: application to the Michoacan, Mexico, earthquake of September 19, 1985, Geophys. Res. Lett., 13, 173-176.

LANGSTON, C.A. and D.V. Helmberger (1975): A procedure for modelling shallow dislocation sources, Geophys. J. R. Astron. Soc., 42, 117-130.

MENKE, W. (1984): Geophysical data analysis (Academic Press, Florida).

NABELEK, J.L. (1984): Determination of earthquake source parameters from inversion of body waves, $\mathrm{Ph}$. D. Thesis, Mass. Inst. of Technol.

Romanowicz, B. and G. SuAREZ (1983): On an improved method to obtain the moment tensor and depth of earthquakes from the amplitude spectrum of Rayleigh waves, Bull. Seismol. Soc. Am., 73, 1513-1526.

Woodhouse, J. H. (1988): The calculation of eigenfrequencies and eigefunctions of the free oscillations of the Earth and the Sun, in Seismological Algorithms, edited by D.J. Doornbos (Academic Press), pp. 321-370. 